\title{
Pilot study: Alternative spliced variants of the Follicle-Stimulating Hormone Receptor gene in Greek women undergoing In Vitro Fertilisation (IVF)
}

\section{Eudoxia Mamas' ${ }^{1}$, Despoina Mavrogianni' ${ }^{2}$, Rami Raouasnte², Spyros Karkatzoulis², Emmanouela Liokari ${ }^{2}$, Petros Drakakis ${ }^{1}$, Dimitrios Loutradis ${ }^{1}$}

${ }^{1} 1^{\text {st }}$ Department of Obstetrics \& Gynecology, Alexandra General Hospital, Medical School of National and Kapodistrian University of Athens, Athens, Greece

${ }^{2}$ Molecular Biology Laboratory, IVF Unit, $1^{\text {st }}$ Department of Obstetrics \& Gynecology, Alexandra General Hospital, Medical School of National and Kapodistrian University of Athens, Athens, Greece

Corresponding Author

Eudoxia Mamas, 26, Riga Feraiou st, N. Psichiko, 15451, Athens, Greece, Tel.: +30 6932429800, e-mail: eudoxia.mamas@gmail.com

\section{Abstract}

Assisted Reproduction Technology (ART) has proven to be a valuable tool for infertile couples. Unfortunately, no perfect ovarian stimulation protocol has been designed and ovarian response shows great variability among women. Pharmacogenomics aims at detecting genetic markers so as to individualize protocols in order to maximize ovarian response to treatment. Follicle Stimulation hormone receptor (FSHR) mutations and single nucleotide polymorphisms have been extensively studied. Splice variants of the FSHR have been detected in women undergoing in vitro fertilisation (IVF). This study aims to determine the presence FSH splice variants in Greek women undergoing IVF. RNA was extracted from cumulus cells from 35 women and analysed by real time PCR. Splice variants were detected by gel electrophoresis. Three cases of deletion of exon 9 and 2 cases of insertion of intron 8 were detected in our study group. No association between the presence of splice variants and response to ovarian stimulation was detected. Two live births were detected one in each variant group. Even though these two types of splice variant detected do not show any clinical correlation it is believed that variants of the FSHR may be associated with poor or high response to exogenous gonadotrophin so further research is necessary.

Key words: Follicle Stimulation Hormone Receptor (FSHR), splice variants, Assisted Reproduction Technology (ART), ovarian stimulation, ovarian response.

\section{Introduction}

FSH is one of the fundamental hormones of mammalian reproductionresponsible for folliculo-

genesis $^{1}$ and spermatogenesis ${ }^{2}$. FSH is a member of the glycoprotein hormone family along with 
LH, TSH and hCG. Each of these hormones share a common $\alpha$ subunit and differ in their unique $\beta$ subunit.FSH regulates ovarian and testicular function through binding with the FSH receptor located in granulosa ${ }^{3}$ and Sertoli cells ${ }^{4}$. The FSH receptor (FSHR) is a G protein-coupled receptor composed of seven transmembrane helices flanked by intracellular and extracellular domains. The intracellular portion which is coupled to a $\mathrm{G}_{\mathrm{s}}$ protein, activates the adenyl cyclase enzyme causing an increase in intracellular cAMP and activation of the cAMP dependent protein kinase pathway ${ }^{5}$. Inactivating mutations of the FSHR gene have been identified in women with primaryor secondary amenorrhea, infertility, polycystic ovary syndrome whereas activating mutations have been linked with ovarian hyperstimulation syndrome ${ }^{6}$.

FSH is one of the key hormones used during ovulation induction. It has been noted however, that the ovarian response to FSH stimulation shows great interindividual variability. Many scientists have throughout the years attempted to identify factors responsible for this variable response. Numerous single nucleotide polymorphisms (SNPs) have been located in the FSHR gene,however only two amino acid substitutions in the coding region (p.307Thr/ Ala rs6165 and p.68-Asn/Ser rs6166)have been extensively studied regarding theirrelationship with ovarian response and IVF outcome ${ }^{7,8}$.

Following cloning of the FSHR cDNA, it became apparent that alternatively spliced transcripts were co-expressed in the gonads of various species including the cattle, rat, mouse, sheep and human ${ }^{9,10}$. In humans, deletion of exons 6 and 9 and insertion of part of intron 8 has been identified in infertile men $^{11}$. For women undergoing IVF, del exon 2 has been linked with poor ovarian response whereas, del exon 6 with a high ovarian response. Deletion of exon 9 and insertion of $102 \mathrm{bp}$ of intron 8 has also been reported in this group 5 .

\section{Material and Methods}

\section{Patient selection and ovulation induction}

Patients were recruited from the IVF clinic of Alexandra Hospital, Athens, Greece. The research protocol was approved by the Ethics Committee of Alexandra Hospital and all participants signed an informed consent before enrolling to the study.

Demographic characteristics (age, Body Mass Index, duration and cause of infertility) and baseline hormonal profile (day 2 FSH, LH and PRL) were recorded before ovarian stimulation.

Gonadotrophin dose, duration and response to ovarian stimulation, number of oocytes and fertilised oocytes were monitored during the treatment cycle for each patient.

\section{Ovulation induction}

For agonists cycles, treatment was initiated following pituitary suppression using intranasal Buserelin spray (Superfact; Hoechst, Frankfurt, Germany). Downregulation and resulting ovarian suppression was confirmed with E2 levels $(<40 \mathrm{pg} / \mathrm{ml})$ and ultrasound scan (absence of follicular cysts). In antagonist cycles, treatment was initiated on day 2 or 3 of the cycle after ultrasonographic evaluation revealed the absence of ovarian cysts. Stimulation with 150 - 300 IU/day of either recombinant FSH (Gonal-F, Serono, Geneva, Switzerland) or in combination with hMG (Merional, IBSA, Lugano, Switzerland) was then initiated. Serum E2 levels were measured on day 5 and then daily after day 8. Follicle growth and endometrial thickness was assessed daily by ultrasound from day 9. In cases of abnormal ovarian response the dose of rFSH was adjusted. When two or more follicles reached $18 \mathrm{~mm}$ or greater in diameter with adequate serum E2 level increase, hCG (10,000IU, Pregnyl; Organon, Oss, Netherlands) was administered for triggering final oocyte maturation. Oocytes were collected 36 hours later by transvaginal ultrasound guided ovarian puncture. 


\section{RNA extraction and CDNA preparation}

Following oocyte retrieval, oocytes were stripped of the surrounding cumulus oocyte complexes (COCs) with mechanical manipulation in hyaluronidase and COCs were collected by aspirating the hyaluronidase solution in a $1.5 \mathrm{ml}$ tube and centrifuged at 1000 $\mathrm{x} g$ for 10 minutes. RNA was extracted using the RNeasy Micro Kit (Qiagen, Valencia, CA, USA) according to manufacturer's instructions. RNA was then reverse transcribed using $8 \mu \mathrm{l}$ dNTP mix, $10 \mu \mathrm{l}$ nuclease free water, $4 \mu$ l oligo dT Primer (Ambion, Austin, TX, USA), $2 \mu$ l ribonuclease inhibitor and $2 \mu \mathrm{l}$ M-MLV reverse transcriptase (Invitrogen, Life Technologies, Carlsbad, CA, USA). The reaction was carried out in Mastercycler (Eppendorf, Hamburg, Germany) using the following conditions: $80^{\circ} \mathrm{C}$ for $3 \mathrm{~min}, 42^{\circ} \mathrm{C}$ for $60 \mathrm{~min}$ and $92^{\circ} \mathrm{C}$ for $10 \mathrm{~min}$. The cDNA products were then stored at $-20^{\circ} \mathrm{C}$. Quality of cDNA was evaluated by amplification of G6PD gene (LightMix Kit human G6PD, TIB MOLBIOL, Berlin, Germany) using the LightCycler 480 II (Roche, Basel, Switzerland).

\section{Detection of splice variants with RT-PCR}

cDNA was used for the detection of splice variants of the FSHR gene through reverse transcription PCR (RT-PCR). The primers for the primary PCR were obtained by TIB MOLBIOL as follows: FSHR.1F, 5'GGA GGT TTT TCT CTG CAA ATG CAG3' and FSHR.2R, 5'CAT ACC CTT CAA AGG CAA GGA CTG3'. The RTPCR program was $50^{\circ} \mathrm{C}$ for $30 \mathrm{~min}, 95^{\circ} \mathrm{C}$ for $15 \mathrm{~min}$, 10 cycles: $92^{\circ} \mathrm{C}$ for $30 \mathrm{sec}, 65^{\circ} \mathrm{C}$ for $20 \mathrm{sec}, 72^{\circ} \mathrm{C}$ for $3 \mathrm{~min}$; 20 cycles: $92^{\circ} \mathrm{C}$ for $20 \mathrm{sec}, 55^{\circ} \mathrm{C}$ for $15 \mathrm{sec} 72^{\circ} \mathrm{C}$ for 5 min. Nested PCR for FSHR was performed using the same conditions and the combination of primers: FSHR.9F, 5'ATG GCC CTG CTC CTG GTC TC3' with FSHR.10R, 5'CAA ACT CAG TGT ACG TCA TGT C3' and FSHR.2F, 5' ATC CCA GCC ATT GCT GTG CCT TTG3' with FSHR.8R, 5'TTA GTT TTG GGC TAA ATG AC3'. The PCR products were analysed on $2 \%$ agarose gel.

\section{Results}

A total of 35women with infertility due to malefactor, tubal factor or unexplained infertility were included in the study. The women were of Greek origin, aged $28-40$ years old with a normal basal serum FSH level (2 - 10IU/lt).

RNA was extracted from cumulus cells, cDNA was synthesized and G6PD expression was measured using real time PCR.Amplification of FSHR cDNA was then carried out using nested PCR. Transcripts of the receptor were detected 30 out of 35 patients (85\%). These samples were further analysed to assess the presence ofalternatively spliced variants of the FSHR.

Ovarian response to ovulation induction was determined by the number of retrieved oocytes and the oocyte maturation rate. Pregnancy rates were also recorded.

Splice variants were identified in $17 \%$ of the patients (5 of 30); three patients showing deletion of exon 9 (Figure 1) and two patients showing insertion of intron 8. Table 1 shows the baseline characteristics among the 30 women with normal FSHR transcripts and the 5 women

withFSHR splice variants. These five patients showed similar response to ovulation induction irrespective of the induction protocol used (two

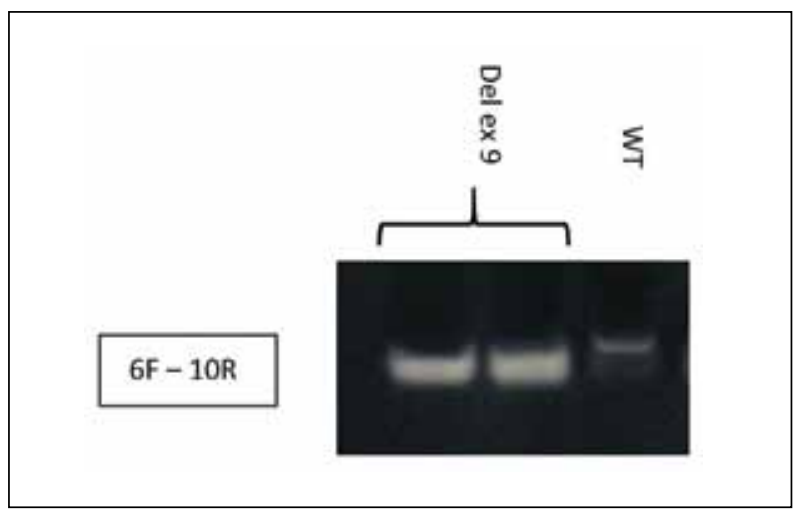

Figure 1. RT-PCR products from women showing deletion of exon 9 FSHR variant. 
Table 1. Comparison of clinical characteristics among patients.

\begin{tabular}{lcc}
\hline & $\begin{array}{c}\text { Normal FSHR } \\
\text { transcripts }\end{array}$ & $\begin{array}{c}\text { FSHR Splice } \\
\text { Variants }\end{array}$ \\
$\mathbf{n}$ & 30 & 5 \\
Age (years) & 32 & 34 \\
BMI (kg/ & 25.3 & 24.8 \\
$\mathbf{m} 2)$ & & \\
FSH (IU/ & 5,5 & 6,1 \\
$\mathbf{m L})$ & & \\
$\mathbf{L H}(\mathbf{I U} / \mathbf{m L})$ & 5,9 & 4,5 \\
$\mathbf{A M H}(\mathbf{n g} /$ & 2,6 & 3,0 \\
$\mathbf{m L})$ & &
\end{tabular}

Data shown as a mean. FSHR: Follicle stimulating Hormone Receptor; BMI: Body Mass Index; FSH: Follicle Stimulating Hormone; LH: Leutinizing Hormone; AMH: Anti-Müllerian Hormone

withagonist and three with antagonist protocol). All five patients showed a good ovarian response with more than 8 oocytes retrieved and a mean oocyte maturation rate of $81 \%$. Two live births were recorded in the group of patients where splice variants were identified (Table 2).

\section{Discussion}

ART has proven to be a valuable option in couples suffering from infertility. Following extensive research it has been deduced that no definite (demographic, anthropometric, hormonal) characteristic can predict a woman's ovarian response to exogenous gonadotrophins ${ }^{12}$.Some women show resistance to exogenous gonadotrophins also knownhyporesponse, a term included in the new POSEIDON classification of 'low prognosis' women undergoing ART ${ }^{13}$. This has urged researchers to look for variants that could affect ovarian response to stimulation ultimately allowing scientists to design patient specific ovarian stimulation protocols. This pharmacogenomic approach if applied in ART will ultimately minimise poor response rates or excessive ovarian response in ovulation induction protocols. The available research is however very limited proving that the field of pharmacogenomics in ART is still immature.

In this study, alternatively spliced variants of the FHSR were analysed in Greek women undergoing IVF treatment. Based on the limited available literature, we focused on locating deletion of exon 2, 6 and 9 as well as insertion of $102 \mathrm{bp}$ in intron 8 in this group of women ${ }^{5}$.

Of the FSHR splice variants investigated in this study, only two have been shown to have clinical significance in women undergoing IVF in North America and Turkey. Deletion of exon 2 has been linked to poor ovarian response to exogenous gonadotrophins. This FSHR variant when co-expressed with the WT receptor shows an inability to generate intracellular increases in cyclic adenosine monophosphate (cAMP) ${ }^{14}$. Karakaya et al, also located deletion of exon 3 in one woman who was a poor responder. On the other hand, women with deletion of exon 6 appeared to have an increased ovarian response ${ }^{5}$.

Deletion of exon 2 and 5 has been located in the mouse FSHR ${ }^{15}$ and deletion of exons 4,5 and 9 in the cattle ${ }^{16}$. These species however, show a different normal ovarian function with multiple dominant

Table 2. Clinical characteristics of patients with FSHR splice variants.

\begin{tabular}{|c|c|c|c|c|c|c|c|c|c|}
\hline $\begin{array}{l}\text { FSH variant } \\
\text { detected }\end{array}$ & $\begin{array}{l}\text { Reason } \\
\text { for IVF }\end{array}$ & Age & $\begin{array}{c}\text { Day } 2 \text { FSH } \\
\text { (IU/ml) }\end{array}$ & $\begin{array}{c}\text { Days of } \\
\text { stimulation }\end{array}$ & $\begin{array}{l}\text { E2 day } \\
\text { of hCG }\end{array}$ & Oocytes & $\begin{array}{l}\text { Maturation } \\
\text { rate }\end{array}$ & Protocol & $\begin{array}{c}\text { Pregnancy } \\
\text { outcome }\end{array}$ \\
\hline \multirow[t]{3}{*}{ Del exon 9} & TUBAL & 33 & 4,79 & 12 & 2108 & 9 & 78 & Antagonist & LB \\
\hline & MALE & 38 & 4,7 & 9 & 2355 & 8 & 88 & Agonist & neg \\
\hline & MALE & 33 & 5,7 & 9 & 1568 & 12 & 83 & Antagonist & neg \\
\hline \multirow[t]{2}{*}{ Ins intron 8} & UNEX & 33 & 7,71 & 8 & 4195 & 12 & 75 & Agonist & LB \\
\hline & MALE & 32 & 7,64 & 10 & 2028 & 11 & 81 & Antagonist & neg \\
\hline
\end{tabular}


follicles developing in each cycle compared to only one in the human, emphasizing the fact that variation in FSHR splicing may be necessary among species.

Research has also shown that deletion of exon 6 and 9 and insertion of intron 8 can be found in infertile men ${ }^{11}$.

This is the first study to investigate alternative splicing of the FSHR in Greek women undergoing IVF treatment. The presence of FSHR spliced variants in women undergoing IVF varied from 35\% in North American ${ }^{5}$ women to $8 \%$ in Turkey ${ }^{14}$, showing great variation among populations. Researches however believe that a clinical association does exist especially in the group of women with deletion of exon 2.

In our study, alternatively spliced variants were detected in $16 \%$ of patients; three cases of deletion of exon 9 and two cases of insertion in intron 8 (Figure 2). These five women were

found to have similar characteristics (BMI, basal FSH level) as well as ovarian response to exogenous gonadotrophins (assessed through E2 level on the day of hCG administration, the total number of oocytes retrieved and oocyte maturation rate). In an attempt to gather more information, pregnancy rates were also evaluated even though it was not one of our primary end points. Once again the data were inconclusive as two live births were recorded, one in a woman showing deletion of exon 9 and one with insertion of intron 8.

Even though the data in this study are limited, it is believed that the presence of a variant FSHR may be associated with an abnormal response to FSH. All the previous published studies have a common finding, a low detection rate of FSHR splice variants. This is also the case in our study with a variant detection rate of $16 \%$. One possible hypothesis to explain this is that alternative splicing of the FSHR may be mediated by the action of hCG administered for oocyte maturation. Screening of a greater number of subjectsis necessary in order to define the association of FSHR spliced variants with ovarian response and female infertility. Future research should specifically aim at women who show a poor response to ovarian stimulation as a connection has been made by Gerasimova et al and Karakaya et al in the past ${ }^{5,14}$. Collecting cumulus cells from poor responders however, may be challenging as a great number of these cycles are cancelled and moreover a limited number of oocytes is usually retrieved in these cycle therefore yielding lower numbers of cumulus cells. As a result, the experimental method must be tailored in such a way to optimise results even though the amount RNA is very limited. It is believed that technological advances will be able to overcome these difficulties and allow easier identification of FSHR variants in an attempt to formulate a genetic profile prior to

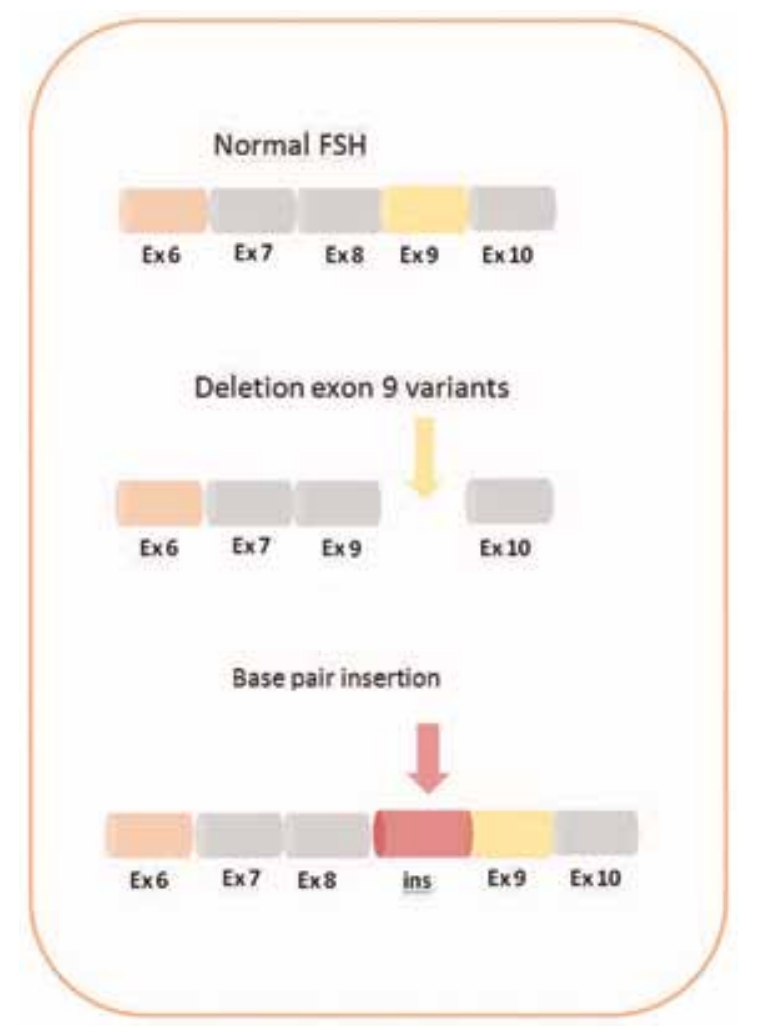

Figure 2. Schematic representation of FSH variants detected in the study. 
ovulation induction. Our research is ongoing and we are confident that in the near future we will have more insight on the effect of FSHR splice variants especially in poor responders.

\section{Conclusion}

FSHR splice variants may prove to play a role in how a woman responds to ovarian stimulation. Unfortunately, the rate of detection of FSHR variants is low but it is believed that a deletion of exon 2 may be highly associated with poor response to exogenous gonadotrophins. The variants detected in this study including Greek women undergoing IVF did not show any clinical correlation to ovarian response which is in accordance to what Gerasimova et al have previously published ${ }^{5}$. Further research is necessary to investigate a larger group of subjects aiming more specifically at poor responders in Greece. This will enable us to determine whether a correlation really exists so as to utilise specific FSHR variants for optimising ovarian stimulation protocols.

\section{References}

1. Richards JS, Midgley AR. Protein hormone action: a key to understanding ovarian follicular and luteal cell development. Biol Reprod 1976;14:82-94.

2. Nieschlag E, Simoni M, Grommol J, Weinbauer GF. Role of FSH in the regulation of spermatiogenesis: clinical aspects. Clin Endocrinol 1999;51:139-146.

3. Camp TA, Rahal JO, Mayo KE. Cellular localization and hormonal regulation of follicle-stimulating hormone and luteinizing hormone receptor messenger RNAs in the rat ovary. Mol Endocrinol 1991;5:1405-1417.

4. Heckert LL, Griswold MD. Expression of folliclestimulating hormone receptor mRNA in rat testes and Sertoli cells. Mol Endocrinol 1991;5:670-677.

5. Gerasimova T, Thanasoula MN, Zattas D, Seli E, Sakkas D, Lalioti MD. Identification and in vitro characterization of follicle stimulating hormone (FSH) receptor variants associated with abnormal ovarian response to FSH. J Clin Endocrinol Metab 2010;95:529-536.

6. Desai SS, Roy BS, Mahale SD. Mutations and polymorphisms in FSH receptor: functional implications in human reproduction. Reproduction 2013;146:235-248.

7. Loutradis D, Patsoula E, Minas V, Koussidis GA, Antsaklis A, Michalas S, Makrygiannakis A. FSH receptor gene polymorphisms have a role for different ovarian response to stimulation in patients entering IVF/ICSI-ET programs. J Assist Reprod Genet 2006;23:177-184.

8. Achrekar SK, Modi DN, Desai SK, Mangoli VS, Mahale SD. Follicle-stimulating hormone receptor polymorphism (Thr307Ala) is associated with variable ovarian response and ovarian hyperstimulation syndrome in Indian women. Fertil Steril2009;91:432-439.

9. Themmen AP, Kraaij R, Grootegoed JA, Themmen APN. Regulation of gonadotropin receptor gene expression. Mol Cel Endocrinol 1994;100:15-19.

10. Yaron Y, Schwartz D, Evans MI, Lessing JB, Rotter V. Alternatively spliced mRNA transcripts encoding the extracellular domain of the FSH receptor gene. J Reprod Med 1998;42:435-438.

11. Song GJ, Park YS, Lee YS, Lee CC, Kang IS. Alternatively spliced variants of the follicle-stimulating hormone receptor gene in the testis of infertile men. Fertil Steril 2002;77:499-504.

12. Conforti A, Cariati F, Vallone R, Alviggi C, de Placido G. Individualization of treatment in controlled ovarian stimulation: myth or reality? Biochim Clin 2017;41:294-305.

13. Poseidon Group (Patient - Oriented Strategies Encompassing Individualized Oocyte Number), Alviggi C, Andersen CY, Buehler K, Conforti A, De Placido G, Esteves SC, Fischer R, Galliano D, Polyzos NP, Sunkara SK, Ubaldi FM, Humaidan P. A 
new more detailed stratification of low responders to ovarian stimulation: from a poor ovarian response to a low prognosis concept. Fertil Steril 2016;105:1452-1453.

14. Karakaya C, Guzeloglu-Kayisli O, Hobbs RJ, Gerasimove T, Uyar A, Erdem M, Oktem M, Erdem A, Gumuslu S, Ercan D, Sakkas D, Comizzoli P, Seli E, Lalioti MD. Follicle-stimulating hormone receptor (FSHR) alternative skipping of exon 2 or 3 affects ovarian response to FSH. Mol Hum Reprod 2014;20:630-643.

15. O'Shaighnessy PJ, Marsh P, Dudley K. Follicle-Stimulating hormone receptor mRNA in the mouse ovary during post-natal development in the normal mouse in the adult hypogonadal (hpg) mouse: structure of alternative transcripts. Mol Cel Endocrinol 1994;101:197-201.

16. Rajapaksha WR, Rovertson L, O'Shaughnessy PJ. Expression of follicle stimulating hormone-receptor mRNA alternative transcripts in bovine granulosa cells during luteinisation in vivo and in vitro. Mol Cell Endocrinol 1996;120:25-30.

Received 30-7-2019

Revised 3-9-2019

Accepted 9-9-2019 